\title{
Monitoring the setting of calcium-based bone cements using pulse-echo ultrasound
}

\author{
M. NILSSON \\ Biomaterials Lab, Department of Orthopaedics, Biomedical Centre, C12, SE-221 84 Lund, \\ Sweden \\ J. CARLSON* \\ EISLAB, Department of Computer Science and Electrical Engineering, Luleå University of \\ Technology, SE-971 87 Luleå, Sweden \\ E. FERNANDEZ, J. A. PLANELL \\ Research Centre in Biomedical Engineering, Biomaterials Division, Department of Materials \\ Science and Metallurgy, Polytechnical University of Catalonia, Avda Diagonal 647, 08028- \\ Barcelona, Spain \\ E-mail: Johan.Carlson@sm.luth.se
}

We present a new technique, based on pulse-echo ultrasound, for monitoring the entire setting process of injectable bone cement. This research has been motivated by the lack of satisfying standards. The main problem with existing standards is the subjectivity, which leads to poor reproducibility. Because of this the results are not comparable between different research groups.

A strong advantage with the proposed technique is that if low-intensity ultrasound is used, it provides a non-destructive analysis method. Once the cement paste has been applied to the measurement cell, no manipulation is needed throughout the entire setting process. The problem of the ultrasound affecting the setting of certain cement materials has been investigated, and solutions are discussed. The propagation of ultrasound is temperaturedependent, and therefore a technique for automatic compensation for temperature variations is discussed briefly.

The testing was performed on $\alpha$-calcium sulfate hemihydrate (CSH) and mixtures of CSH and $\alpha$-tricalcium phosphate ( $\alpha$-TCP). The results show that the acoustic properties of the cement are strongly correlated with the setting time, the density, and the adiabatic bulk modulus. The measured initial and final setting times agree well with the Gillmore needles standard. An important difference compared to the standards, is that the technique presented here allows the user to follow the entire setting process on-line.

(C) 2002 Kluwer Academic Publishers

\section{Introduction}

Quantifying the setting process is important when working with injectable bone substitutes [1] for bone defect healing. It is essential to know the strength and the setting time of the material, to decide when and how it should be injected into the bone.

There are currently two standardized methods to study the hardening process, the Gillmore needles method (ASTM C266-89) [2] and the Vicat needle (ASTM C191-92) [3]. The idea of both methods is to visually examine the surface of the cement samples to decide if the material has reached the setting time, i.e. if no mark can be seen on the surface of the cement. The visual examination makes the test methods subjective with large individual variations. Some examples of such variations in the resulted setting time have been reported.
Norian SRS has been said to set at $27 \mathrm{~min}$ [4], $22 \pm 1 \mathrm{~min}$ [5] or $8.5 \pm 0.5 \mathrm{~min}$ [6], depending on the researcher. Similar variations exist for Cementek (34 min [4], $36 \mathrm{~min}$ [5] and $17 \pm 1 \mathrm{~min}$ [6]) and BoneSource (19 min [4] and 20-25 min [7]).

To overcome this problem we introduce an ultrasonic method that allows us to continuously follow the setting reaction. This method gives more information about the evolution of the setting process than the standardized methods do. Furthermore, this analysis method is objective.

Other advantages with an ultrasonic test method is that other properties than the setting time could be derived from the setting curve, e.g. the adiabatic bulk modulus.

In clinical applications it is important to know when the cement material is hard enough to close the wound

* Author to whom all correspondence should be addressed. 
without damaging it. According to Driessens et al. [8], the cement material is hard enough when it can withstand a static pressure of $5 \mathrm{MPa}$ without leaving a visible mark on the surface. This corresponds to the heavy needle in the Gillmore needles test, and the time obtained is defined as the final setting time. The desired time for the final setting time should be between 10 and $15 \mathrm{~min}$ in clinical applications [9]. Most of the cement materials available on the market do not fulfill this requirement. Sarda et al. [10] suggest, using rheology measurements, that the material probably reaches a sufficient strength to withstand the pressure during wound closing long before the measured final setting time.

Recently, Viano et al. [11] presented an ultrasonic technique for the characterization of bone cement based on polymethylmethacrylate (PMMA). Their method uses the broadband attenuation of pulsed ultrasound (BUA) and the speed of sound within the cement to determine when the cement is set. They use two ultrasound transducers in a through-transmission setup. The BUA and speed of sound both vary dramatically during the setting process, and this shows that the acoustic properties of the materials can be used to characterize different types of bone cements. Although the BUA is very sensitive to variations in viscoelastic properties of the material, there is no immediate connection between the BUA value and the actual mechanical properties of the material.

The purpose of the work presented in this paper is to show that an ultrasonic pulse-echo technique can be used to follow certain properties of the cement, on-line, during the setting reaction. The idea is verified with experiments on calcium sulfate hemihydrate $(\mathrm{CSH}) \alpha$-tri-calcium phosphate $(\alpha$-TCP). The results show that determining when the cement is set is possible even with a fairly simple setup. If, however, exact measurements of the physical properties of the material is needed, some additional computation and calibration must be done in order to compensate for temperature fluctuations during the measurement. This was done for some of the experiments presented in this paper. Experiments were also made to study if and how the transmitted ultrasound pulse affects the setting process.

\section{Materials and methods}

\subsection{Materials}

Experiments were made on two different cements, one which was pure $\alpha$-calcium sulfate hemihydrate (CSH), and another which was a mix of CSH and $\alpha$-tricalcium phosphate $(\alpha$-TCP).

For the first experiment series, $30 \mathrm{~g}$ of $\mathrm{CSH}$ powder (Bo Ehrlander AB, Gothenburg, Sweden) was mixed with an aqueous solution of $2.5 \%$ (by weight; wt \%) of $\mathrm{Na}_{2} \mathrm{HPO}_{4}$ at a liquid-to-powder (L/P) ratio of $0.32 \mathrm{ml} \mathrm{g}^{-1}$, during $1 \mathrm{~min}$, to form a paste. During the setting CSH hydrates into CSD following the reaction in Equation 1.

$$
\mathrm{CaSO}_{4} \cdot \frac{1}{2} \mathrm{H}_{2} \mathrm{O}+\frac{3}{2} \mathrm{H}_{2} \mathrm{O} \rightarrow \mathrm{CaSO}_{4} \cdot 2 \mathrm{H}_{2} \mathrm{O}
$$

For the second experiment series, the cement paste consisted of $80 \mathrm{wt} \%$ of $\alpha$-TCP, produced as described in

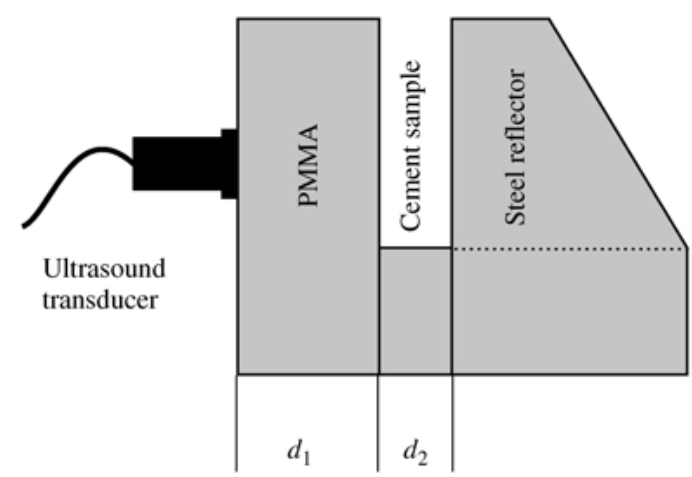

Figure 1 Device for ultrasonic pulse-echo measurements used in the experiments. The distances $d_{1}$ and $d_{2}$ are 20 and $15 \mathrm{~mm}$, respectively.

a previous study [12], and $20 \mathrm{wt} \%$ of CSH. The same liquid as above was used to form the paste.

The mixing time was $1 \mathrm{~min}$. Thereafter, the paste was baked into the measurement cell (see Fig. 1), making sure that it completely covered the ultrasound transducer. Data collection started 3 min after the mixing started, and the cell was immersed in water at $37^{\circ} \mathrm{C}, 5$ min after the mixing started. Data were then collected every 2 min until the cement was set. For CSH this meant measuring for $2 \mathrm{~h}$, while for the $\mathrm{CSH} / \alpha$-TCP mix, the experiment continued for $24 \mathrm{~h}$. For all measurements a $2 \mathrm{MHz}$ ultrasound transducer was used to transmit short-duration pulses (approx. $3.5 \mu$ s) into the cement. The time interval between the transmitted pulses was set using the pulserepetition frequency (PRF) settings of the pulser/receiver (Panametrics, model P5800). For the CSH experiments, the pulser/receiver was set to a PRF of $10 \mathrm{kHz}$, and an excitation energy of $100 \mu \mathrm{J}$. This meant sending a $100 \mu \mathrm{J}$ impulse to the transducer. The transducer converts this electrical energy to a mechanical sound wave, propagating through the medium. The actual amplitude of the outgoing sound wave is, however, unknown, since the exact conversion from electrical to mechanical energy not known.

For the CSH/ $\alpha$-TCP mix, three different measurement signals were used to determine if the PRF and/or the energy of the ultrasound pulses had any effect on the setting of the materials. The pulser/receiver was used to excite the ultrasound transducer with either a $25 \mu \mathrm{J}$ or a $100 \mu \mathrm{J}$ impulse. The first two measurements were made using the $100 \mu \mathrm{J}$ setting, with a PRF of either $80 \mathrm{~Hz}$ or $10 \mathrm{kHz}$. The third measurement was with the $25 \mu \mathrm{J}$ setting and a PRF of $80 \mathrm{~Hz}$. The purpose of this was to check if there was any detectable difference in the results when sending strong pulses at a high PRF, or weaker pulses at a low PRF.

For each individual experiment, one pulse-echo measurement was made with only water in the measurement cell. These measurements serve as reference when calculating the mechanical properties described in the following section.

\subsection{Setting time with Gillmore needles}

A reference experiment was made in compliance with the Gillmore needles standard in order to compare the new ultrasonic technique with the existing standard. $5 \mathrm{~g}$ of $\mathrm{CSH}$ powder was mixed with $1.6 \mathrm{ml}$ of the $\mathrm{Na}_{2} \mathrm{HPO}_{4}{ }^{-}$ 
solution ( $\left.\mathrm{L} / \mathrm{P}=0.32 \mathrm{mlg}^{-1}\right)$ during $1 \mathrm{~min}$ to form a paste. To perform the setting time test it was then put in molds and tested according to the Gillmore needle standard [2]. The initial setting time (I) is defined as the time when a $0.3 \mathrm{MPa}$ static pressure does not leave a visible print on the surface of the cement. The final setting time $(F)$ is the corresponding time for a static pressure of $5 \mathrm{MPa}$.

No Gillmore needles tests were made for the $\alpha-\mathrm{TCP} /$ CSH cement.

\subsection{Acoustic measurement principle}

The principle of the method presented in this paper is to measure the acoustic impedance at the interface between a material with known acoustic properties and the cement. This together with the speed of sound in the cement is then used to calculate density and adiabatic bulk modulus of the cement. In this section the details of the measurement principle are described in detail.

When a sound wave encounters a boundary between two materials with different acoustic properties, part of the acoustic energy will be reflected back towards the transmitter, and part of the energy will continue into the second medium. How much of the acoustic energy that is reflected back depends on the difference in specific acoustic impedance between the two layers. The acoustic impedance, $\mathrm{Z}\left(\mathrm{Pa} \cdot \mathrm{s} \mathrm{m}^{-3}\right)$ can be related to an analog electrical system in a similar way as voltage is related to sound pressure and current is related to particle or volume velocity. The specific acoustic impedance, $z$, of a material has the unit pressure/particle velocity $\left(\mathrm{Pa} \cdot \mathrm{s} \mathrm{m}^{-1}\right)$ and is very useful in calculations involving transmission and reflection of sound waves [13]. In the rest of this paper the term acoustic impedance is referring to the specific acoustic impedance.

Fig. 1 shows the measurement cell used in the experiments. An ultrasound transducer was mounted on the surface of a plexiglass (PMMA) buffer. This idea was first introduced by Lynnworth [14] as a method to measure density of liquids. An overview of other pulseecho setups can be found in van Deventer [15].

In our setup, the transducer is first used to transmit a short ultrasound pulse with a center frequency of $2 \mathrm{MHz}$. For a sound velocities of 2500 and $3500 \mathrm{~m} \mathrm{~s}^{-1}$, this corresponds to a wavelength of 1.25 and $1.75 \mathrm{~mm}$, respectively $(\lambda=c / f)$. The same transducer is then used as a receiver to record the reflected echoes. The electrical pulses recorded with the transducer are then sampled at a sampling frequency of $200 \mathrm{MHz}$ and transferred to a computer. Fig. 1 shows the measurement cell, and Fig. 2 shows an example of the received signal when the measurement cell is filled with $\mathrm{CSH}$ cement. The first echo, $x_{1}(t)$ is from the PMMA/cement interface and the second echo, $x_{2}(t)$, comes from the interface between the cement and the steel reflector.

The ratio of the amplitude of the first echo $x_{1}(t)$ to a corresponding echo measured in a calibration experiment in pure water gives the acoustic impedance of the cement. The time of flight of the second echo, $x_{2}(t)$, and the thickness of the cement sample gives the speed of sound in the cement. These two properties can then be used to determine the density and the adiabatic bulk

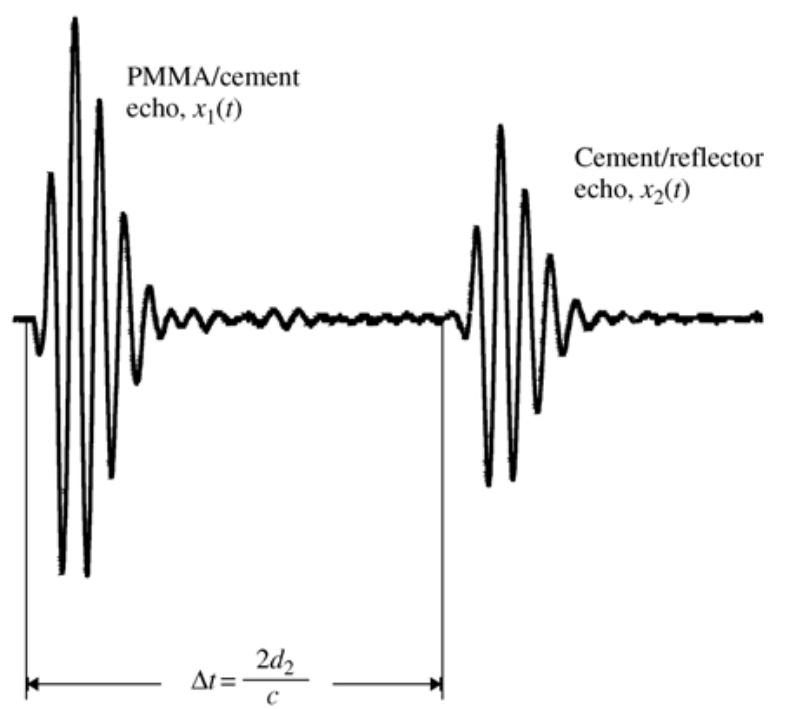

Figure 2 Reflected pulse from PMMA/cement interface, $x_{1}(t)$ and reflected pulse from cement/reflector interface, $x_{2}(t)$, respectively. The time delay between is denoted $\Delta t$.

modulus, $\beta$, of the cement. The adiabatic bulk modulus can be written as [16]

$$
\beta=C-\frac{4 G}{3}
$$

where the compression wave modulus, $C$, and the shear wave modulus $G$ are given by

$$
\begin{gathered}
C=\lambda+2 \mu \\
G=\mu
\end{gathered}
$$

and $\lambda$ and $\mu$ are the Lamé constants of the solid material. In the current setup, only a compression wave transducer is used, and therefore it is only possible to determine the bulk modulus, and not $C$ and $G$ in Equation 2. Being a combination the compression modulus and the shear modulus, it is still an interesting measure of the mechanical strength of the material. In order to determine both the shear and the compression modulii it is necessary to measure both the shear wave velocity and the compression wave velocity. This requires the use of both compression wave and shear wave ultrasound transducers.

When the ultrasound transducer is excited with an electrical impulse, it begins to oscillate, which gives rise to a sound pressure wave. Because the relation between the input impulse to the amplitude of the emitted sound wave is not known, it is necessary to determine this experimentally. In the calibration measurement, when the measurement cell only contains water, the amplitude of the first echo, coming from the PMMA/water interface, is given by

$$
A_{w}\left(T_{0}\right)=A_{0} R_{p, w} \mathrm{e}^{-\alpha\left(T_{0}\right) 2 d_{1}}
$$

where $T_{0}$ is the temperature, $A_{0}$ is the (unknown) amplitude of the transmitted pulse, $R_{p, w}$ is the reflection coefficient (see Equation 11) for the PMMA/water boundary, $d_{1}$ is the thickness of the PMMA, and $\alpha\left(T_{0}\right)$ is the attenuation coefficient for PMMA at temperature 
$T_{0}$. Solving for the amplitude of the transmitted pulse, we obtain

$$
A_{0}=\frac{A_{w}\left(T_{0}\right)}{R_{p, w} \mathrm{e}^{-\alpha\left(T_{0}\right) 2 d_{1}}}
$$

When the measurement cell is filled with cement paste, the corresponding expression for the reflected echo amplitude becomes

$$
\begin{aligned}
A_{c}\left(T_{1}\right) & =A_{0} R_{p, c} \mathrm{e}^{-\alpha\left(T_{1}\right) 2 d_{1}} \\
& =A_{w}\left(T_{0}\right) \frac{R_{p, c}}{R_{p, w}} \mathrm{e}^{-2 d_{1}\left(\alpha\left(T_{1}\right)-\alpha\left(T_{0}\right)\right)}
\end{aligned}
$$

The reflection coefficient $R_{p, c}$ is defined as the amount of reflected acoustic energy at the PMMA/cement interface. From Equation 8 this is given by,

$$
R_{p, c}=\frac{A_{c}}{A_{w}} R_{p, w} \mathrm{e}^{2 d_{1}\left(\alpha\left(T_{1}\right)-\alpha\left(T_{0}\right)\right)}
$$

For the special case, when the temperatures are the same for the two measurements, this simplifies to

$$
R_{p, c}=\frac{A_{c}}{A_{w}} R_{p, w}
$$

Since the attenuation coefficient $\alpha(T)$ is a function of temperature [17], it is obvious from Equation 9 that the temperature of the PMMA has to be either constant or measured, in order to obtain an accurate measurement of the reflection coefficient $R_{p, c}$. It was shown in [17] that the speed of sound in PMMA varies linearly with temperature for a wide temperature range. Calibrating the measurement cell at a known temperature thus enables us to estimate the temperature of PMMA, by using the changes in transit-time for the first echo, $x_{1}(t)$. This was done by using a standard cross-correlation technique in combination with a more precise method for subsample time-delay estimation [18]. The estimated temperature can then be used to determine the densities and sound velocities in water and PMMA, respectively.

To determine $R_{p, c}$ (Equation 10), we need to know $R_{p, w}$ and the amplitude ratio. The reflection coefficient [13] can also be expressed as

$$
R_{p, w}=\frac{z_{w}-z_{p}}{z_{w}+z_{p}}
$$

where $z_{w}$ and $z_{p}$ are the known acoustic impedances of water [19] and PMMA [20], respectively. These properties are all temperature dependent, but known [21] and the $T$ is therefore dropped from the equation, for simplicity. At $37^{\circ} \mathrm{C}, z_{\mathrm{w}}=1.4852 \times 10^{6} \mathrm{~Pa} \cdot \mathrm{s} \mathrm{m}^{-1}$ and $z_{p}=3.2007 \times 10^{6} \mathrm{~Pa} \cdot \mathrm{s} \mathrm{m}^{-1}$.

The amplitude ratio in Equation 10 is calculated by first taking the discrete Fourier transform (DFT) of the sampled pulses, and then calculating the amplitude ratio $A_{\mathrm{c}} / A_{\mathrm{w}}$ at the center frequency of the transducer, in this case at $2 \mathrm{MHz}$.

Once the reflection coefficient $R_{p, c}$ has been determined, the acoustic impedance of the cement sample can be calculated as

$$
z_{c}=z_{p} \frac{1+R_{p, c}}{1-R_{p, c}}
$$

In order to determine the speed of sound in the cement, the two echoes $x_{1}(t)$ and $x_{2}(t)$ (see Fig. 1) are cross correlated. The maximum of the cross correlation corresponds to the time-shift $\Delta t$ between the two pulses. Since the propagation distance, $d_{2}$, of the pulse is known, the speed of sound is $c=2 d_{2} / \Delta t$.

From the speed of sound, $c$, and the acoustic impedance, $z_{c}$ (Equation 12) of the cement, the density, $\rho$, and the adiabatic bulk modulus, $\beta$, are given by

$$
\begin{aligned}
& \rho=\frac{z_{c}}{c} \\
& \beta=c^{2} \rho
\end{aligned}
$$

\section{Results}

Using the pulse-echo technique described in the previous section it is possible to measure the acoustic impedance and the speed of sound in the cement sample, on-line, during the setting of the cement.

Two series of experiments were made. The first was to study the setting of CSH. This material sets in less than an hour, and therefore it was possible to keep the temperature variations small enough for Equation 10 to be valid.

The second experiment series was planned in order to study how the technique performs on a mixture of $\mathrm{CSH}$ and $\alpha$-TCP, an injectable bone substitute [1]. In this case we observed larger temperature fluctuations, and therefore the measured echo amplitudes were corrected for the temperature-dependent attenuation in the PMMA part of the measurement cell in Fig. 1.

\subsection{Setting of $\mathrm{CSH}$}

Fig. 3 shows the acoustic impedance at the PMMA/ cement interface as a function of the setting time. The figure clearly shows that when the cement is set, the rate of change of the acoustic impedance decreases. The (I) and $(\mathrm{F})$ times obtained with the Gillmore needles method are also marked in the figure, and they were determined to be 17 and $25 \mathrm{~min}$, respectively.

Fig. 4 shows the speed of sound in the cement as a function of the setting time. The speed of sound was

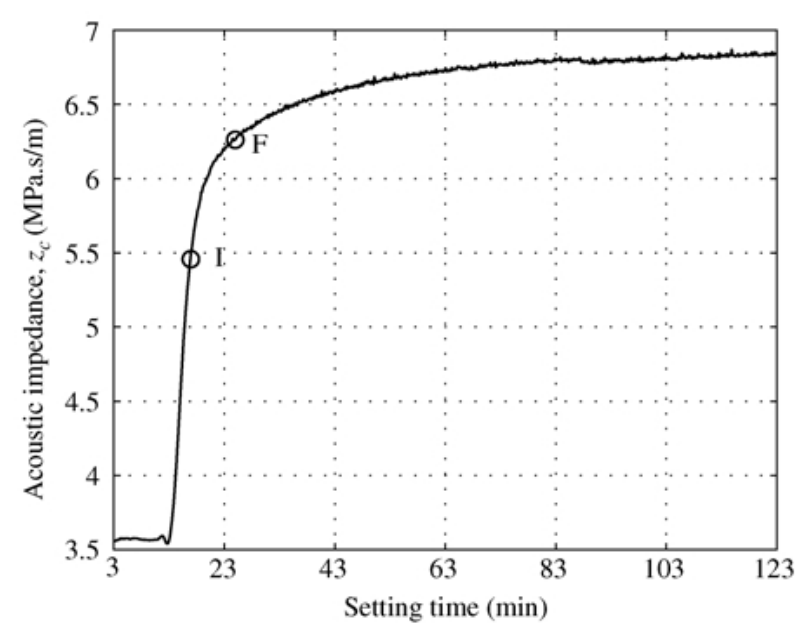

Figure 3 Acoustic impedance of CSD as a function of the setting time. 


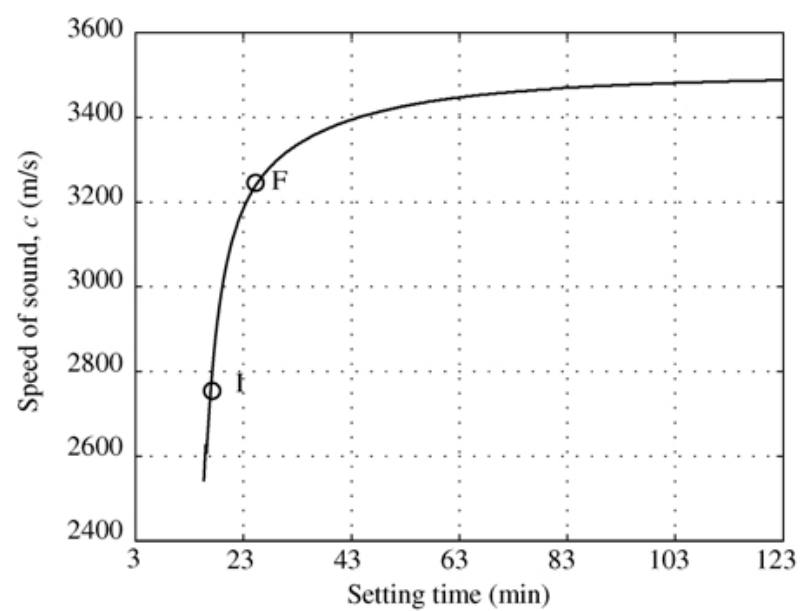

Figure 4 Speed of sound in CSD as a function of the setting time.

obtained by cross-correlating the echo from the steel reflector with the echo from the PMMA/cement interface. In the early stages of the setting process, most of the sound energy was absorbed by the cement and thus the second echo was very weak. For this time interval $(0-$ $15 \mathrm{~min}$ ) it was therefore not possible to determine the sound velocity accurately.

The speed of sound reaches a final value of approximately $3500 \mathrm{~m} \mathrm{~s}^{-1}$. This could be compared to the speed of sound in corresponding value in water $\left(1495 \mathrm{~m} \mathrm{~s}^{-1}\right.$, at $\left.37^{\circ} \mathrm{C}\right)$, in PMMA $\left(2700 \mathrm{~m} \mathrm{~s}^{-1}\right.$, at $\left.37^{\circ} \mathrm{C}\right)$, and stainless steel $\left(5980 \mathrm{~m} \mathrm{~s}^{-1}\right.$, at $\left.37^{\circ} \mathrm{C}\right)$ [22].

Fig. 5 shows the adiabatic bulk modulus as a function of the setting time. This was calculated from the speed of sound and the acoustic impedance using Equation 14. The adiabatic bulk modulus is a property related to the elasticity and compressive strength of the material. Using only compressional sound waves, it is not possible to completely solve for the different modulii of the material [23]. It is interesting to note that the adiabatic bulk modulus varies from about $1 \mathrm{GPa}$ to about $25 \mathrm{GPa}$. These are in the same order of magnitude as the Young's modulus ( $E$ in Equation 2) of cancellous bone, which is approximately $1 \mathrm{GPa}$, and that of compact bone, which is approximately $30 \mathrm{GPa}$. This is one reason why calcium

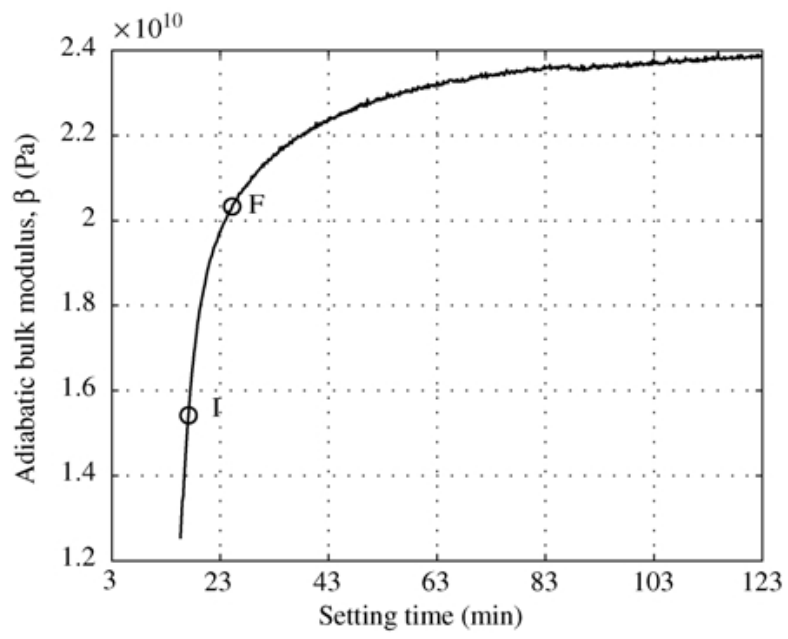

Figure 5 Adiabatic bulk modulus of CSD as a function of the setting time.

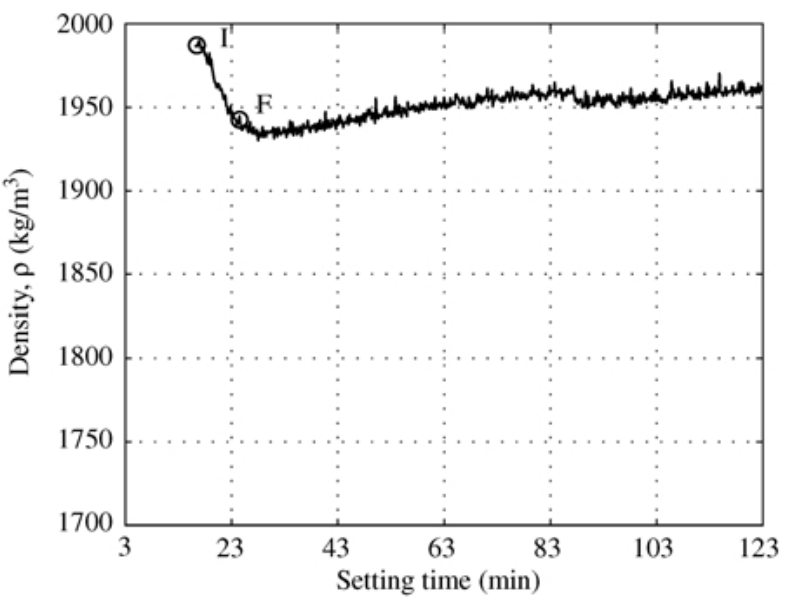

Figure 6 Density of CSD as a function of the setting time.

sulfate-based materials could be good substitutes for damaged bone.

From the measured values of the acoustic impedance and the speed of sound, it is also possible to determine the density of the cement at the PMMA/cement interface. The results are shown in Fig. 6. The figure shows that there is a sudden decrease in density between the (I) and (F) times, measured with the Gillmore needles standard. The slow increase in density in the time interval 20$120 \mathrm{~min}$, was about $1.6 \%$, which is in the same range as the documented shrinkage observed by others [24,25]. The exact shrinkage depends on how the cement is prepared, and under what conditions the setting takes place.

\subsection{Setting of $\alpha$-TCP/CSH}

Fig. 7 shows the acoustic impedance of three different measurements on mixtures of $80 \mathrm{wt} \%$ of $\alpha$-TCP and $20 \mathrm{wt} \% \mathrm{CSH}$. Experiments were made using different settings of the outgoing ultrasound energy, as well as the PRF of the ultrasound, i.e. how often an ultrasound pulse was transmitted into the cement paste. The purpose of these experiments was to examine if the ultrasound had any effect on the setting process.

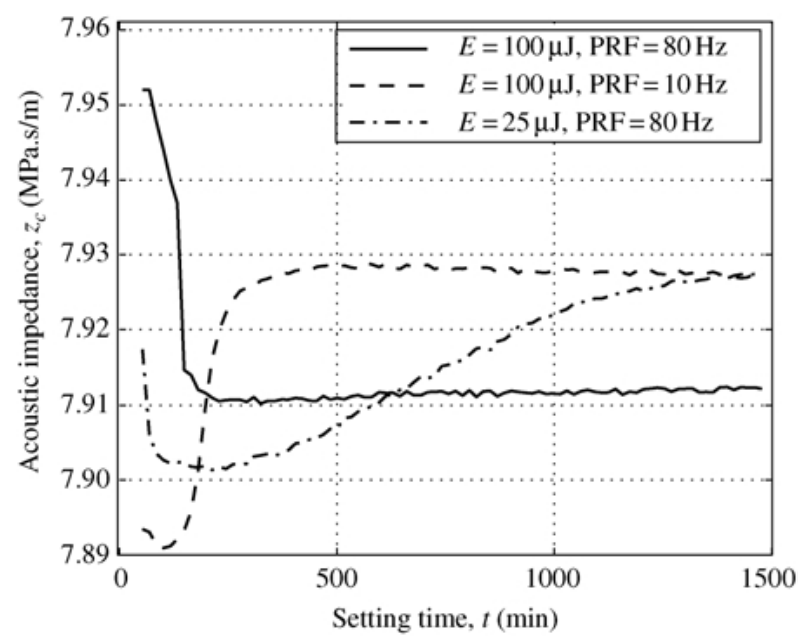

Figure 7 Acoustic impedance of $\alpha$-TCP as a function of the setting time, for three different settings of the acoustic parameters. 
The solid and the dashed lines in Fig. 7 are the results obtained with a high intensity ultrasound pulse $(100 \mu \mathrm{J})$, at a PRF of $80 \mathrm{~Hz}$ and $10 \mathrm{kHz}$, respectively. The dasheddotted line is the result when using low-intensity ultrasound $(25 \mu \mathrm{J})$, at a PRF of $80 \mathrm{~Hz}$. The same $2 \mathrm{MHz}$ as in the CSH experiments were used in these experiments as well.

Because of the high attenuation of sound in the $\alpha$-TCP/ $\mathrm{CSH}$ it was not possible to obtain an accurate estimate of the sound velocity in the cement. Without the speed of sound, it was not possible to determine the adiabatic bulk modulus or density in these experiments, but only the acoustic impedance at the interface between the PMMA and the cement (Equation 12).

The results shown in Fig. 7 indicate that the use of an ultrasound pulse with higher intensity, could accelerate the setting of the material.

\section{Discussion}

There are several reasons why a method that continuously measures the hardening of a cement material is preferred over the existing standards. By following the whole setting process it is possible to observe strength parameters at any time during setting. That would help the surgeon to decide when the material is hard enough to close the patient's wound. If the surgeon is able to close the wound earlier, this is not only good from an economical point of view, but also reduces the risks of medical complications. Since the proposed method is objective, less variation depending on the user is expected compared to the standardized method based on visual examination. Furthermore, using the pulseecho technique, it is also possible to measure other properties about the cement than just the setting time. Looking in more detail at the backscattered sound, it should be possible to assess the porosity of the material. The reflected pulses changes in shape and length if the material becomes porous during setting. This is because when the sound hits cavities and boundaries between different crystals, partial reflections of the pulse within the crystal structure will occur. This effect will then change the shape of the pulse coming back to the transducer, compared to the pulse obtained for a nonporous cement.

The use of longitudinal pressure waves allows the determination of the adiabatic bulk modulus of the material. This modulus is a combination of parameters that characterize the compressive strength and the elasticity. In this setup, where we use the information from the reflected pulse, it is, however, not possible to solve for these parameters. It could be done by slightly modifying the experimental setup to use a combination of longitudinal and shear waves, instead of longitudinal waves alone. An example of this type of setup was proposed by Freemantle and Challis [16] as a technique to monitor curing adhesive.

The results obtained with Gillmore needles agree with the results from the ultrasonic technique. The (I) time (17 min) was found during the fast increase in Figs. 3-5. However, this is the most critical part of the curve where small errors in time change the result significantly. Thus, the results may differ a lot from user to user. The (F) time
( $25 \mathrm{~min}$ ) is found just when the curves attain the plateau, which shows that the $(\mathrm{F})$ time indicates a good value of the time when the final properties of the cement are obtained. After this point no large changes are seen in either of Figs. 3-5.

For the density, the results show a different behavior (Fig. 6). The curve is first decreasing until after approximately $25 \mathrm{~min}$, and then slightly increasing until the end of the experiment. The (I) time is found as the first measurable point in the figure while the $(\mathrm{F})$ time almost corresponds to its lowest point. We cannot determine the speed of sound in the cement at the beginning of the experiment, when the cement is still very wet. Most of the sound energy is absorbed and no echo is obtained from the reflector (the cement/steel interface). Since the density is derived from the speed of sound no data can be presented in the beginning of Fig. 6 . One possible solution to this problem would be to use an ultrasound transducer with lower frequency than $2 \mathrm{MHz}$, because lower frequencies are attenuated less.

The first point observed in the curve is when the attenuation of the cement is sufficiently low to obtain a second echo. This is probably due to a rapid change in the crystal structure in the cement during a short period of time. The minimum of the curve can be associated to a point where the end of the hydration and the beginning of the shrinking process occurs. During shrinkage the density increases slightly, as seen in the figure. The final density, measured after $2 \mathrm{~h}$, differs slightly from theoretical values found in literature [19]. The exact density, however, depends on both the liquid-to-powder ratio as well as if the density was measured in wet or dry conditions.

The experimental results presented for $\mathrm{CSH}$, see Equation 1, clearly show the feasibility of using ultrasound to measure the setting time and also to determine some mechanical properties of the material. For the mixtures of CSH with $\alpha$-TCP, the results indicate that the ultrasound could affect the setting, i.e. the crystallization of the materials. The crystallization process could be disturbed in either crystal formation or crystal growth. This problem has to be overcome if the technique is to be usable in practice. There are several possible solutions to this problem. One is to use ultrasound with lower frequency than $2 \mathrm{MHz}$. Lower frequencies are attenuated less by the cement, which allows the intensity to be decreased, i.e. allows a lower sound pressure. Future research should also examine which ultrasound frequencies that are best suited for this type of materials. Furthermore, the present ultrasonic equipment transmits the pulses continuously, at a certain pulse-repetition frequency (PRF) as opposed to transmitting the sound only when data is collected. This means that, even for the lowest PRF setting, an ultrasound pulse was transmitted through the cement 80 times per second. Modifying the setup to overcome this would most likely reduce the effect on the material significantly.

\section{Conclusions}

In this paper we have presented an ultrasonic pulse-echo technique that can be used to measure the setting time, 
the density, and the adiabatic modulus of calcium sulfate based bone cement.

The method is easy to use and the testing can be made throughout the entire setting process without having to move or otherwise manipulate the cement sample. As opposed to the existing standards, where the initial setting period is measured at two points, the proposed technique shows the development during the entire setting process.

Preliminary experimental results on $\alpha$-TCP based cements indicate that using an ultrasound pulse with too high energy, could affect the setting process. This is a problem that has to be solved in future research, and some possible solutions are presented in this paper.

Most important, the results obtained with the ultrasonic technique are re-producible and completely objective, which makes it possible to compare results between different researchers.

Moreover, the acoustic method could be implemented to be operated at the operation theater as a way to avoid batch-to-batch differences of cement material, if necessary.

\section{Acknowledgment}

The authors thank the European Commission for funding through programs of Objective 1 Norra Norrland (1131623-2001), the European Commission project: Training Site HPMTCT200000003, the Margit \& Folke Pehrzon Foundation (Madrid, Spain), Medical Faculty at Lund University, The Swedish Research Council (proj. 09509), and Stiftelsen för or bistånd åt rörelsehindrade i Skåne. The authors would also like to express their gratitude towards Dr. Jan van Deventer for his valuable comments, Prof. Jerker Delsing and Prof. Anders Grennberg at Luleå University of Technology, and Prof. Lars Lidgren at the Department of Orthopaedics, Lund University Hospital for supporting this work.

\section{References}

1. M. NILSSON, E. FERNÁNDEZ, S. SARDA, L. LIDGREN and J. A. Planell, Key. Eng. Mater. 218-220 (2002) 365.

2. ASTM C266-89, in "Annual Book of ASTM Standards", Vol. 04.01: Cement, Lime, Gypsum (Philadelphia, 1993) pp. 189-191.

3. ASTM C191-92, in "Annual Book of ASTM Standards", Vol. 04.01: Cement, Lime, Gypsum (Philadelphia, 1993) pp. 158-160.
4. R. WENZ, M. G. BOLTONG and F. C. M. DRIESSENS, in "Transactions Vol. I of the VIth World Biomaterials Congress", edited by Society for Biomaterials, (Hawaii, May 2000), p. 339.

5. F. C. M. DRIESSENS, I. KHAIROUN, M. G. BOLTONG and R. WENZ, in "Transactions Vol. III of the VIth World Biomaterials Congress', edited by Society for Biomaterials, (Hawaii, May 2000), p. 1107.

6. M. B OHNER, Eur. Spine J. 10 (2001) 114.

7. B ONES OURCE, http://www.tarma.es/tarma/BoneSource.html (9 February 2002).

8. F. C. M. DRIESSENS, J. A. Planell, M. G. BOLTONG, I. KHAIROUN and M. P. GINEB RA, Proc. Instn. Mech. Engrs. 212 (1998) 427.

9. M. P. GINEBRA, E. FERnÁndez, M. G. BOLTONG, O. BERMÚdEZ, J. A. PLANELL and F. C. M. DRIESSENS, Clin. Mater. 17 (1994) 99.

10. S. SARDA, E. FERNÁNDEZ, J. LLORENS, S. MARTÍNEZ, M. NilsS ON and J. A. PlANELl, Mater. Sci: Mater. in Med. 12 (2001) 905 .

11. A. M. ViAno, J. A. AUWARter, J. Y. RHO and B. K. HOFFMEIS TER, J. Biomed. Mater. Res. 56 (2001) 593.

12. M. P. GINEBRA, E. FERNÁNDEZ, E. A. P. DE MAEYER, R. M. H. VERBEECK, M. G. BOLtONG, J. GinebRA, F. C. M. DRIESSENS and J. A. PLANELL, J. Dent. Res. 76 (1997) 905.

13. L. E. KINSLER, A. R. FREY, A. B. COPPENS and J. V. S ANDERS, "Fundamentals of Acoustics"' 3rd edn (John Wiley, New York, 1982).

14. L. C. LYNNWORTH, in "Ultrasonics Mass Flowmeter for Army Aircraft Engine Diagnostics. National Technical Information Services"' (Springfield, 1973).

15. J. VAN DEVENTER, Ph.D Thesis, Luleå University of Technology (2001).

16. R. J. FREEMANTLE and R. E. CHALlis, Meas. Sci. Technol. 9 (1998) 1291.

17. J. VAN DEVENTER and J. DELSING, IEEE Trans. Ultras. Ferroelect., Freq. Contr. 48 (2001), 675.

18. A. GRENNBERG and M. SANDELL, ibid. 41 (1994) 588.

19. D. R. LIDE (editor), in "CRC Handbook of Chemistry and Physics", 79th edn (CRC Press, 1998).

20. W. WUNDERLICH, “Polymer Handbook' (Wiley-Interscience, New York, 1989).

21. J. VAN DEVENTER, T. LÖFQVIST and J. DELSING, IEEE Trans. Ultras. Ferroelect., Freq. Contr. 47 (2000), 1014.

22. G. W. C. KAYE and T. H. LABY, "Tables of Physical and Chemical Constants", (Longman, London, 1995).

23. Y. C. FUNG, "Foundations of solid mechanics", 11th edn (Prentice-Hall, New Jersey, 1965) pp. 127-131.

24. A. J. LEWRY and J. Williamson, J. Mater. Sci. 29 (1994) 5524.

25. K. D. JøRGENSEN and A. S. POSNER, J. Dent. Res. 38 (1959) 491.

Received 24 May

and accepted 29 May 2002 